\title{
Applications of the Theory of Continuous Oil Film Lubrication of a Radial Sliding Bearing for Three Combined Conditions
}

\author{
Jingyuan $\mathrm{Li}^{1} \&$ Zifeng $\mathrm{Li}^{1}$ \\ ${ }^{1}$ Petroleum Engineering Department, Yanshan University, Qinhuangdao 066004, Hebei, China \\ Correspondence: Zifeng Li, Petroleum Engineering Department, Yanshan University, Qinhuangdao 066004, \\ Hebei, China. E-mail: zfli@ysu.edu.cn
}

Received: January 22, 2016

doi:10.5539/apr.v8n2p22
Accepted: February 1, 2016 Online Published: February 15, 2016

URL: http://dx.doi.org/10.5539/apr.v8n2p22

\begin{abstract}
The theory of lubrication of a radial sliding bearing is extended to three cases: the shaft neck rotation and bush rotation bearing, the rotating load bearing, and the floating sleeve bearing. For the bearing with rotating shaft neck and rotating bush, fixing the observer at the bushing can enable the determination, in a simple and more accurate manner, of the bearing capacity of the bearing with the rotating load. As long as a rotating load exists, whirl exists; if the shaft rotation speed is 2 times the load rotation speed, then the eccentricity reaches the maximum.
\end{abstract}

Keywords: radial sliding bearing, rotating load, floating sleeve, whirl

\section{Introduction}

The theory of the lubrication of a radial sliding bearing introduces theories for a fixed bush, shaft neck rotation and/or revolution (Wen \& Huang, 2008; Sfyris \& Chasalebris, 2012; Santos et al., 2012; Kasolanga et al., 2012), but is inadequate for modeling the other complex conditions (Wang et al., 2004; Li et al., 2005; Wang et al., 2010; Tian et al., 2011; Tian et al., 2012; Yu et al., 2013). In this paper, the theory of continuous oil film lubrication of a radial sliding bearing will be extended to three cases: the shaft neck rotation and bush rotation bearing, the rotation load bearing, and the floating sleeve bearing.

\section{Basic assumptions}

1) The volume force is ignored.

2) The inertial force is ignored.

3) The fluid has no sliding on the interface.

4) No pressure is changed in the direction of the thickness of the lubricating film.

5) The radius of curvature of the rotation component surface is relatively large compared with the oil film thickness.

6) The lubricant is a Newtonian fluid.

7) The compressibility of the lubricant is ignored.

8) The flow is laminar flow, and there is no eddy flow or turbulent flow in the oil film.

9) The viscosity along the thickness of the lubricating film is not changed.

10) The influence of pressure and temperature on the oil viscosity is ignored.

Although the author recognizes that neglecting the lubricant compressibility can lead to the obvious error or mistake that the shaft neck center and the bush center lie on the same horizontal line while carrying a vertical load, the author is not yet able to deduce the lubrication theory for a compressible lubricant.

\section{Existing Theory of Lubrication of an Infinitely Long Radial Sliding Bearing Dynamic}

Figure 1 shows the shaft neck with a fixed load rotating in the bush. In this figure, the bush is fixed, and the shaft neck rotates clockwise. 


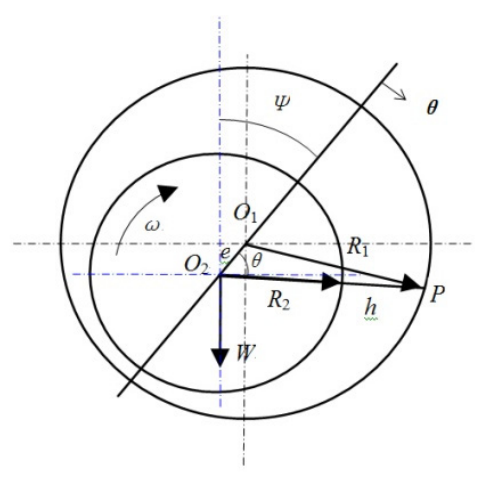

Figure 1. Bush and shaft neck positions

The Reynolds equation and the Sommerfeld boundary conditions are used to solve the dynamic pressure distribution in an infinitely long bearing, and then, the bearing capacity is calculated (Wen \& Huang, 2008).

3.1 Dynamic Pressure Distribution (Wen \& Huang, 2008)

The Reynolds equation of the infinitely long bearing represented by polar coordinates is

$$
\frac{\mathrm{d} p}{\mathrm{~d} \theta}=6 U \eta R \frac{h-\bar{h}}{h^{3}}
$$

where $U=R \omega$. Because the gap is very small, $R \approx R_{1} \approx R_{2}$.

Because

$$
h=c(1+\varepsilon \cos \theta)
$$

where $c=R_{1}-R_{2} ; \varepsilon=e / c$. After integration

$$
p=\frac{6 \eta \omega R^{2}}{c^{2}}\left[\int \frac{\mathrm{d} \theta}{(1+\varepsilon \cos \theta)^{2}}-\frac{\bar{h}}{c} \int \frac{\mathrm{d} \theta}{(1+\varepsilon \cos \theta)^{3}}\right]+c_{1}
$$

Equation (3) can be integrated using the conversion $\cos \gamma=\frac{\varepsilon+\cos \theta}{1+\varepsilon \cos \theta}$, and based on the Sommerfeld boundary conditions, which is when $\theta=0$ and $\theta=2 \pi, p=0$, we obtain $c_{1}=0$ and $\bar{h}=\frac{2 c\left(1-\varepsilon^{2}\right)}{2+\varepsilon^{2}}$, resulting in the following

$$
p(\theta)=\frac{6 \eta \omega R^{2}}{c^{2}} \cdot \frac{\varepsilon(2+\varepsilon \cos \theta) \sin \theta}{\left(2+\varepsilon^{2}\right)(1+\varepsilon \cos \theta)^{2}}
$$

Anti-symmetric to $\theta=\pi$, i.e., negative pressure area is formed in the divergence area.

The absolute pressure of the entire region can be greater than zero by adjusting the static pressure value to avoid evaporation.

\subsection{Load (Wen \& Huang, 2008)}

The load component $W_{y}$ in the direction vertical to line of centers is

$$
W_{y}=W \sin \psi=\int_{0}^{2 \pi} L R p \sin \theta \mathrm{d} \theta
$$

Using partial integration of the formula, we obtain

$$
W \sin \psi=L R\left[-\left.p \cos \theta\right|_{0} ^{2 \pi}+\int_{0}^{2 \pi} \frac{\mathrm{d} p}{\mathrm{~d} \theta} \cos \theta d \theta\right]=L R \int_{0}^{2 \pi} \frac{\mathrm{d} p}{\mathrm{~d} \theta} \cos \theta \mathrm{d} \theta
$$


Substituting $\frac{\mathrm{d} p}{\mathrm{~d} \theta}$ and after performing the integral calculation, we obtain

$$
W \sin \psi=\frac{12 \pi \eta \omega R L(R / c)^{2} \varepsilon}{\left(2+\varepsilon^{2}\right)\left(1-\varepsilon^{2}\right)^{1 / 2}}
$$

Similarly, we can determine the load component $W_{x}$ in the direction of line of centers, i.e.

$$
W_{x}=W \cos \psi=\int_{0}^{2 \pi} L R p \cos \theta \mathrm{d} \theta=0
$$

Because $W \neq 0, \cos \psi=0$, i.e., $\psi=\frac{\pi}{2}$.

Therefore, the axis track solved by the Sommerfeld boundary condition is a straight line vertical to the direction of load $W$. Apparently, this conclusion is not true. When considering the compressibility of the lubricant, the shaft neck center should be at the lower left of the bush center.

\section{Dynamic Pressure Distribution and Bearing Capacity when Shaft Neck and Bush are Simultaneously Rotating}

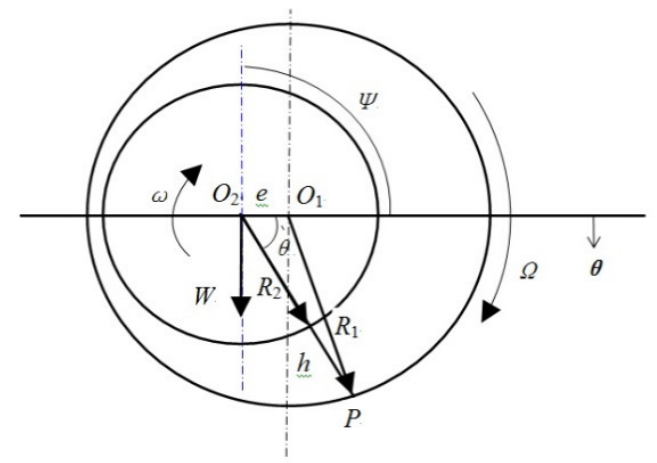

Figure 2. The shaft neck and bush are simultaneously rotating

In Figure 2, the shaft neck and bush are simultaneously rotating. Now that the shaft neck center and the bush center are on the same horizontal line based on the above calculation, these two centers are drawn on the same horizontal line. The other quantities are the same as those in Figure 1.

Both the dynamic pressure and the load produced in the following two conditions can be proven to be the same: (1) when the shaft neck does not rotate, the bush rotates with the angular speed of $\omega ;(2)$ when the bush does not rotate, the shaft neck rotates with the angular speed of $\omega$. Therefore, the dynamic pressure and the bearing capacity when the shaft neck and bush rotating at the same time are the algebraic sum of these two simple conditions.

\subsection{Dynamic Pressure Distribution}

$$
p(\theta)=\frac{6 \eta(\omega+\Omega) R^{2}}{c^{2}} \cdot \frac{\varepsilon(2+\varepsilon \cos \theta) \sin \theta}{\left(2+\varepsilon^{2}\right)(1+\cos \theta)^{2}}
$$

4.2 Load

$$
W=\frac{12 \pi \eta(\omega+\Omega) R L(R / c)^{2} \varepsilon}{\left(2+\varepsilon^{2}\right)\left(1-\varepsilon^{2}\right)^{1 / 2}}
$$

As shown in Equation (8) and Equation (9), when the angular speed $\Omega$ of the bush and the angular speed $\omega$ are equal in value but opposite in directions, both the dynamic pressure and the bearing capacity are zero. 


\section{Dynamic Pressure Distribution and Bearing Capacity of a Bearing with a Rotating Load}

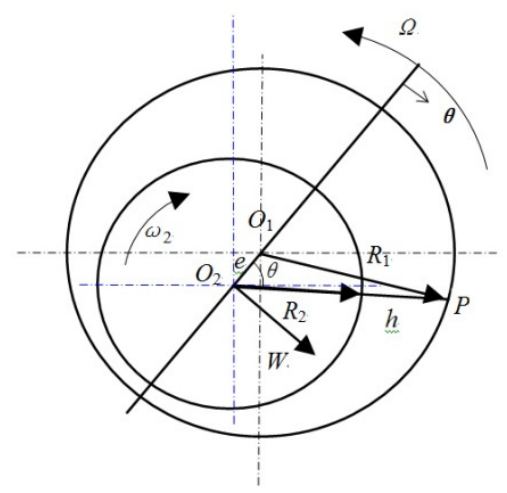

Figure 3. Rotating load bearing

Figure 2 shows the situation in which the shaft neck does not revolve, the shaft neck and bush rotates, and the observer does not move relative to the load. If the observer stands on the bush, then it becomes the rotating load bearing. At this time, the entire figure in Figure 2 rotates with $-\Omega$, as shown in Figure 3. In Figure 3, the bush does not rotate, the angular speed of shaft neck is $\omega_{2}=\omega-\Omega$, and the rotary angular speed of the load is $-\Omega$. Because the pressure distribution and bearing capacity do not change with the observer position, substituting $\omega=\omega_{2}+\Omega$ into Equation (8) and Equation (9), we can obtain the dynamic pressure distribution and the load of the rotating load bearing. In this figure, the bush is fixed, and the shaft neck rotates clockwise and revolves in the anti-clockwise direction.

\subsection{Dynamic Pressure Distribution}

$$
p(\theta)=\frac{6 \eta\left(\omega_{2}+2 \Omega\right) R^{2}}{c^{2}} \cdot \frac{\varepsilon(2+\varepsilon \cos \theta) \sin \theta}{\left(2+\varepsilon^{2}\right)(1+\varepsilon \cos \theta)^{2}}
$$

5.2 Load

$$
W=\frac{12 \pi \eta\left(\omega_{2}+2 \Omega\right) R L(R / c)^{2} \varepsilon}{\left(2+\varepsilon^{2}\right)\left(1-\varepsilon^{2}\right)^{1 / 2}}
$$

The result of Equation (11) is the same as that of the Equation (4-64) in the literature (Wen and Huang, 2008), but the deduction process in this paper is simple and convenient and does not require the precondition of $e / R<<2 \cos \theta$.

As shown in Figure 3 and Equation (11), as long as there is a rotating load, vortex motion of the shaft neck occurs, which is the inevitable result of the rotating load. The vortex motion frequency is exactly the same as that of the rotating load; when the load rotation direction is the same as that of the shaft neck and the rotation speed of the shaft neck is twice of that of the load, the eccentricity reaches the maximum value.

\section{Dynamic Pressure Distribution and Bearing Capacity of the Floating Sleeve Bearing}

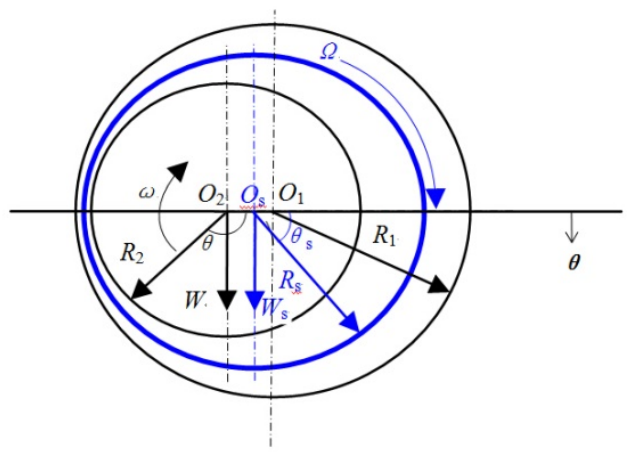

Figure 4. Floating sleeve bearing 
Figure 4 shows one floating sleeve bearing. The bush is fixed. The shaft neck rotates clockwise. Between the bush and the shaft neck, there is a floating sleeve rotating clockwise.

\title{
6.1 Dynamic Pressure Distribution
}

1) Between the bush and the floating sleeve

$$
p_{s}\left(\theta_{s}\right)=\frac{6 \eta \Omega R_{1}^{2}}{c_{s}^{2}} \cdot \frac{\mathcal{E}_{s}\left(2+\varepsilon_{s} \cos \theta_{s}\right) \sin \theta_{s}}{\left(2+\varepsilon_{s}^{2}\right)\left(1+\varepsilon_{s} \cos \theta_{s}\right)^{2}}
$$

where $c_{s}=R_{1}-R_{s}$ and $\varepsilon_{s}=e_{s} / c_{s}$.

2) Between the floating sleeve and the shaft neck

$$
p(\theta)=\frac{6 \eta(\omega+\Omega) R_{2}^{2}}{c_{2}^{2}} \cdot \frac{\varepsilon_{2}\left(2+\varepsilon_{2} \cos \theta\right) \sin \theta}{\left(2+\varepsilon_{2}^{2}\right)\left(1+\varepsilon_{2} \cos \theta\right)^{2}}
$$

where $c_{2}=R_{s}-R_{2}$ and $\varepsilon_{2}=e_{2} / c_{2}$.

$6.2 \mathrm{Load}$

1) Between the bush and the floating sleeve

$$
W_{s}=\frac{12 \pi \eta \Omega R_{1} L\left(R_{1} / c_{s}\right)^{2} \varepsilon_{s}}{\left(2+\varepsilon_{s}^{2}\right)\left(1-\varepsilon_{s}^{2}\right)^{1 / 2}}
$$

2) Between the floating sleeve and the shaft neck

$$
W=\frac{12 \pi \eta(\omega+\Omega) R_{2} L\left(R_{2} / c_{2}\right)^{2} \varepsilon_{2}}{\left(2+\varepsilon_{2}^{2}\right)\left(1-\varepsilon_{2}^{2}\right)^{1 / 2}}
$$

When the shaft neck load $W$, angular speed $\omega$ of the shaft neck, liquid viscosity $\eta$, and geometrical size of the bearing are given, there are four parameters to be solved for: eccentricity $\varepsilon_{\mathrm{s}}$ of the floating sleeve, eccentricity $\varepsilon_{2}$ of the shaft neck inside the floating sleeve, angular speed $\Omega$ of the floating sleeve and load $W_{s}$. Considering $W=W_{s}$, there are still three equations but four unknown quantities. Therefore, either the angular speed $\Omega$ of the floating sleeve is given or a supplementary equation should be established (Wang et al., 2004).

\section{Conclusions}

1) The theory of continuous oil film lubrication of a radial sliding bearing was extended to three conditions: both the bush and the shaft neck rotating; the load rotating; and a sliding bearing with a floating sleeve.

2) The bearing capacity of the bearing with rotating load can be determined in a simple and accurate manner when the observer is fixed on the bush of the bearing with bush and shaft neck both rotating.

3) The shaft neck will cause vortex motion as long as there is a rotating load. The maximum eccentricity appears when the load rotation direction is the same as that of the shaft neck and the rotation speed of the shaft neck is twice that of the load.

\section{Acknowledgment}

The paper is supported by the National Natural Science Foundation of China (Grant No. 51374183 and 51490653).

\section{Nomenclature}

\author{
$c$ average gap \\ $c_{1}$ integral constant \\ $c_{2}$ average gap between the shaft neck and the floating sleeve \\ $c_{s}$ average gap between the floating sleeve and the bush \\ $e$ eccentric distance \\ $e_{2}$ eccentric distance $O_{1}-O_{\mathrm{s}}$ of the shaft neck inside the floating sleeve
}


$e_{\mathrm{s}}$ eccentric distance $O_{1}-O_{\mathrm{s}}$ of the floating sleeve

$h$ clearance

$\bar{h}$ constant

$L$ bearing length

$O_{1}$ bush center

$\mathrm{O}_{2} \quad$ shaft neck center

$O_{S} \quad$ floating sleeve center

$P \quad$ one point on the bush face

$p \quad$ dynamic pressure

$R$ radius

$R_{1} \quad$ bush radius

$R_{2} \quad$ shaft neck radius

$R_{S} \quad$ floating sleeve radius

$U$ speed of the shaft neck surface relative to the fixed bush surface

$W$ load

$W_{s}$ bearing load

$W_{x} \quad$ load component in the direction of the line of centers

$W_{y} \quad$ load component in the vertical line of centers

$\varepsilon$ eccentricity

$\varepsilon_{2}$ eccentricity of the shaft neck inside the floating sleeve

$\varepsilon_{s} \quad$ floating sleeve eccentricity

$\eta \quad$ liquid viscosity

$\theta$ polar angle

$\theta_{s}$ polar angle with the floating sleeve as the polar point

$\psi \quad$ included angle between the load direction and the line of centers

$\Omega \quad$ angular speed of the bush in Figure 2, the load in Figure 3, or the sleeve in Figure 4

$\omega$ angular speed of the shaft neck

SI Units are used.

\section{References}

Kasolanga, S., Ahmada, M. A., Joyceb, R., \& Taib, C. F. M. (2012). Preliminary study of pressure profile in hydrodynamic lubrication journal bearing. Procedia Engineering, 41, 1743-1749. http://dx.doi.org/10.1016/ j.proeng.2012.07.377

Li, Z., Gui, C., Li, Z., \& Sun, J. (2005). Study on dynamic behaviors of variably-loaded shaft-bearing system. Machine Design and Research, 21(1), 12-16. (in Chinese)

Sfyris, D., \& Chasalebris, A. (2012). An exact analytical solution of the Reynolds equation for the finite journal bearing lubrication. Tribology International, 55, 46-58. http://dx.doi.org/10.1016/j.triboint.2012.05.013

Santos, E. N., Blanco, C. J. C., Macedo, E. N., Maneschya, C. E. A., \& Quaresmac, J. N. N. (2012). Integral transform solutions for the analysis of hydrodynamic lubrication of journal bearings. Tribology International, 55, 161-169. http://dx.doi.org/10.1016/j.triboint.2012.03.016

Tian, L., Wang, W., \& Peng, Z. (2011). Dynamic behaviors of a full floating ring bearing supported turbocharger rotor with engine excitation. Journal of Sound and Vibration, 330, 4851-4874. http://dx.doi.org/10.1016/ j.jsv.2011.04.031 
Tian, L., Wang, W., \& Peng, Z. (2012). Effects of bearing outer clearance on the dynamic behaviors of the full floating ring bearing supported turbocharger rotor. Mechanical Systems and Signal Processing, 31, $155-175$. http://dx.doi.org/10.1016/j.ymssp.2012.03.017

Wang, G., Liu, Q., Zheng, J., \& Wu, L. (2004). Investigation of floating-ring journal bearing's working mechanism. Lubrication Engineering, 5, 13-15. (in Chinese)

Wang, Z., Ren, X., Zhang, Y., Kang, Z., \& Yang, Y. (2010). Exploring further basic flow of rotating floating-ring bearing. Journal of Northwestern Polytechnical University, 28(4), 520-524. (in Chinese)

Wen, S., \& Huang, P. (2008). Principles of tribology (3rd Ed., (pp. 84-105). Beijing: Tsinghua University Press. (in Chinese)

Yu, P., Wang, J., \& Wang, Q. (2013). Lubrication performance of short bearing under dynamic load. Lubrication Engineering, 38(7), 12-15, 27. (in Chinese)

\section{Copyrights}

Copyright for this article is retained by the author(s), with first publication rights granted to the journal.

This is an open-access article distributed under the terms and conditions of the Creative Commons Attribution license (http://creativecommons.org/licenses/by/3.0/). 